\title{
Green Tea Epigallocatechin Gallate Exhibits Anticancer Effect in Human Pancreatic Carcinoma Cells via the Inhibition of Both Focal Adhesion Kinase and Insulin-Like Growth Factor-I Receptor
}

\author{
Hoang Anh Vu, ${ }^{1,2}$ Yuuichi Beppu, ${ }^{3}$ Hoang Thanh Chi, ${ }^{2,4}$ Kousuke Sasaki, ${ }^{3}$ \\ Hideaki Yamamoto, ${ }^{3}$ Phan Thi Xinh, ${ }^{2}$ Takashi Tanii, ${ }^{3}$ Yukihiko Hara, ${ }^{5}$ Toshiki Watanabe, \\ Yuko Sato, ${ }^{2}$ and Iwao Ohdomari ${ }^{3}$ \\ ${ }^{1}$ Consolidated Research Institute for Advanced Science and Medical Care (ASMeW), Waseda University, Tokyo 169-8050, Japan \\ ${ }^{2}$ Division of Ultrafine Structure, Department of Pathology, Research Institute of International Medical Center of Japan, \\ Toyama 1-21-1, Shinjuku-ku, Tokyo 162-8655, Japan \\ ${ }^{3}$ Faculty of Science and Engineering, Waseda University, Tokyo 169-8555, Japan \\ ${ }^{4}$ Department of Medical Genome Sciences, Graduate School of Frontier Sciences, The University of Tokyo, Tokyo 108-8639, Japan \\ ${ }^{5}$ Tea Solutions, Food Research Lab, Hara Office Inc, Miyabara, Fujieda City 130-0012, Japan \\ Correspondence should be addressed to Yuko Sato, ysato@ri.ncgm.go.jp
}

Received 10 November 2010; Accepted 29 December 2010

Academic Editor: Gary E. Gallick

Copyright (C) 2010 Hoang Anh Vu et al. This is an open access article distributed under the Creative Commons Attribution License, which permits unrestricted use, distribution, and reproduction in any medium, provided the original work is properly cited.

The exact molecular mechanism by which epigallocatechin gallate (EGCG) suppresses human pancreatic cancer cell proliferation is unclear. We show here that EGCG-treated pancreatic cancer cells AsPC-1 and BxPC-3 decrease cell adhesion ability on micropattern dots, accompanied by dephosphorylations of both focal adhesion kinase (FAK) and insulin-like growth factor-1 receptor (IGF-1R) whereas retained the activations of mitogen-activated protein kinase and mammalian target of rapamycin. The growth of AsPC-1 and BxPC-3 cells can be significantly suppressed by EGCG treatment alone in a dose-dependent manner. At a dose of $100 \mu \mathrm{M}$ which completely abolishes activations of FAK and IGF-1R, EGCG suppresses more than 50\% of cell proliferation without evidence of apoptosis analyzed by PARP cleavage. Finally, the MEK1/2 inhibitor U0126 enhances growth-suppressive effect of EGCG. Our data suggests that blocking FAK and IGF-1R by EGCG could prove valuable for targeted therapy, which can be used in combination with other therapies, for pancreatic cancer.

\section{Introduction}

Worldwide pancreatic cancer is associated with high morbidity, estimated to cause 213,000 deaths annually [1]. Due to late clinical presentation, rapid tumor progression, andresistance to conventional chemotherapeutic agents, the 5 -year survival rate remains less than $5 \%$. The hope for patients with pancreatic cancer comes from an improved understanding of the molecular mechanism underlying the highly aggressive nature of this disease, providing more selective methods for an effective treatment.

Focal adhesion kinase (FAK) is a nonreceptor cytoplasmic tyrosine kinase that plays an important role in several different cell processes including cell proliferation, migration, and survival [2]. The activation of FAK requires autophosphorylation of residue tyrosine 397, upon ligand binding and clustering of intergrin receptors, as well as activation of other cell surface receptors such as EGFR [3]. Importantly, FAK is overexpressed in cancers and inhibition of FAK activity sensitizes cancer cells to apoptosis [4], suggesting that FAK plays a role in carcinogenesis. Very recently, Liu et al. have shown that FAK and insulinlike growth factor 1 receptor (IGF-1R) interact to provide survival signals in pancreatic cancer cells [5]. IGF-1R is one of the major receptor tyrosine kinases to trigger several key molecules for cell proliferation and survival. Overexpression 
of IGF-1R has been detected in many different types of cancers including pancreatic cancer $[6,7]$, making it a potential target for therapy. Indeed, inhibition of IGF-1R by a small molecule kinase inhibitor NVP-AEW541 reduced pancreatic tumor growth [8]. Moreover, TAE226, a novel dual tyrosine kinase inhibitor for FAK and IGF-1R, exhibited anticancer effect in esophageal adenocarcinoma [9] as well as in glioma [10]. In the present study, we have shown for the first time that epigalocatechin galate (EGCG) from natural green tea effectively inhibited phosphorylations of both FAK and IGF-1R in human pancreatic cancer cell lines AsPC-1 and BxPC-3, accompanied by suppression of cell adhesion and proliferation. At a dose of $100 \mu \mathrm{M}$ that already abolished activations of FAK and IGF-1R, EGCG had no effect on either phosphorylations of two major signal transduction pathways believed to play key roles in carcinogenesis, mitogen-activated protein kinase (MAPK) and mammalian target of rapamycin (mTOR), or in causing apoptosis by PARP cleavage analysis. Importantly, MAPK inhibitor enhances EGCG-induced antiproliferative response in cancer cells.

\section{Materials and Methods}

2.1. Cell Lines and Culture Conditions. The human pancreatic cancer cell lines AsPC-1 and BxPC-3, obtained from ATCC (American Type Culture Collection, Manassas, VA, USA), were grown in RPMI supplemented with $10 \%$ fetal bovine serum (FBS; JRH Biosciences, Lenexa, KS, USA), $100 \mathrm{U} / \mathrm{ml}$ penicillin, and $0.1 \mathrm{mg} / \mathrm{ml}$ streptomycin (Nacalai Tesque, Tokyo, Japan) in a humidified incubator of $5 \% \mathrm{CO}_{2}$ at $37^{\circ} \mathrm{C}$.

\subsection{Fabrication of Octadecylsilane Pattern for the Evaluation} of Cell Adhesion. For evaluating the cell adhesion quantitatively, a two-tone organosilane monolayer pattern composed of cell-adhesive region and cell-repellent region was fabricated on glass slides using electron beam lithography. Chemical vapor deposition (CVD) of tetraethylorthosilicate (TEOS) was performed to flatten the Pyrex glass slide. A dual-layer photopolymer film was spin-coated and patterned into an array of $15 \mu \mathrm{m}$ diameter circles by electron beam lithography. The glass slide was then exposed to a vapor of noctadecyltrimethoxysilane (ODS) at $110^{\circ} \mathrm{C}$. By removing the photopolymer film in tetrahydrofuran (THF), the array of holes were formed in the ODS monolayer. After cleaning the glass substrate by sonicating in chloroform and in ethanol for 10 minutes each, the glass surface exposed within the holes was selectively modified with 3 -aminopropyltriethoxysilane (APTES) by immersing the glass substrate in a solution of $2 \%$ APTES in $95 \%$ ethanol/water for 30 minutes. Prior to cell culture, the glass slide was sterilized by immersing the substrate in $70 \%$ ethanol/water for 10 minutes and rinsing in water twice. As previously reported [11], bovine serum albumin (BSA) adsorbed selectively on the ODS monolayer surface prevented the cells from adhering to outside the circles, while the surface modified with APTES prompted the cells to attach to the circles. Thus the cell attachment is limited within the $15 \mu \mathrm{m}$ diameter circles modified with APTES.

AsPC- 1 and BxPC- 3 cells were plated at a density of $0.8 \times 10^{5} \mathrm{cells} / \mathrm{ml}$ in the presence or absence of EGCG onto micropattern coverslips for 24 hours. Cells attaching to single dots were photographed under microscope.

2.3. Reagents. A purified preparation of EGCG was generously provided by Dr. Yukihiko Hara, Mitsui-Norin (Shizuoka, Japan). U0126 (MEK1/2 inhibitor) was purchased from Cell Signaling Technology (Danvers, MA, USA). EGCG and U0126 were dissolved in dimethylsulfoxide (DMSO). The concentration of DMSO was kept under $0.1 \%$ throughout all the experiments to avoid its cytotoxicity.

2.4. Proliferation Assays. For measurement of cell proliferation, cells were seeded in quadruplicate in 96-well plates, in $100 \mu \mathrm{l}$ of culture media in the presence of various concentrations of EGCG. After incubation for 48 hours, $10 \mu \mathrm{l}$ of TetraColor ONE reagent containing tetrazolium monosodium salt (Seikagaku Corporation, Tokyo, Japan) was added to each well and cells were incubated for additional 4 hours. Absorbance at $450 \mathrm{~nm}$ was measured with the Biotrack II plate reader (Amersham Biosciences, Uppsia, Sweden). Results were enumerated as the percentage of the values measured when cells were grown in the absence of EGCG.

For trypan blue exclusion assay, cells were plated in 24well dishes with different concentrations of EGCG for 48 hours, harvested with a brief incubation in $0.05 \%$ trypsin and $0.02 \%$ EDTA, stained with trypan, blue and counted manually using a hemacytometer.

2.5. Western Blot Analysis. Cells were plated onto 6-cm dishes at a density of $2 \times 10^{5} \mathrm{cells} / \mathrm{ml}$ in the presence of various concentrations of EGCG. After incubation for indicated times, cells were collected by trypsinization and washed twice in phosphate-buffered saline (PBS). Cells were then dissolved in a protein lysis buffer containing $5 \mathrm{mM}$ EDTA, $50 \mathrm{mM} \mathrm{NaF}$, $10 \mathrm{mM} \mathrm{Na} \mathrm{H}_{2} \mathrm{P}_{2} \mathrm{O}_{7}, 0.01 \%$ Triton X-100, $5 \mathrm{mM}$ HEPES, $150 \mathrm{mM} \mathrm{NaCl}, 1 \mathrm{mM} \mathrm{Na} 3 \mathrm{VO}_{4}, 1 \mathrm{mM}$ phenylmethylsulfonyl fluoride, and $75 \mu \mathrm{g} / \mathrm{mL}$ aprotinin on ice for 30 minutes with brief vortex every 10 minutes. After centrifugation at $13,000 \mathrm{rpm}$ at $4^{\circ} \mathrm{C}$ for 10 minutes, total cell lysates were collected for western blot analysis as described previously [12].

The following antibodies were obtained from Santa Cruz Biotechnology (Santa Cruz, CA, USA): N-cadherin (sc-7939), FAK (sc-557), IGF-IR $\beta$ (sc-713), ERK1 (sc93), antirabbit IgG- HRP (sc-2317), and antimouse IgGHRP (sc-2031). Antiactin (A2066) was from Sigma (Saint Louis, MO, USA). Phospho-IGF-I-receptor $\beta$ (Tyr1131), phospho-p44/42 Map kinase (Thr202/Tyr204), phospho-Akt (Ser473), phospho-mTOR (Ser2448), caspase-3, caspase-8, and caspase- 9 antibodies were from Cell Signaling Technology. Phospho-FAK (pTyr397) was from ABR Affinity Bioreagents (Golden, CO, USA). Anti-E-cadherin was from BD Biosciences (San Jose, CA, USA). Anti-PARP was from 

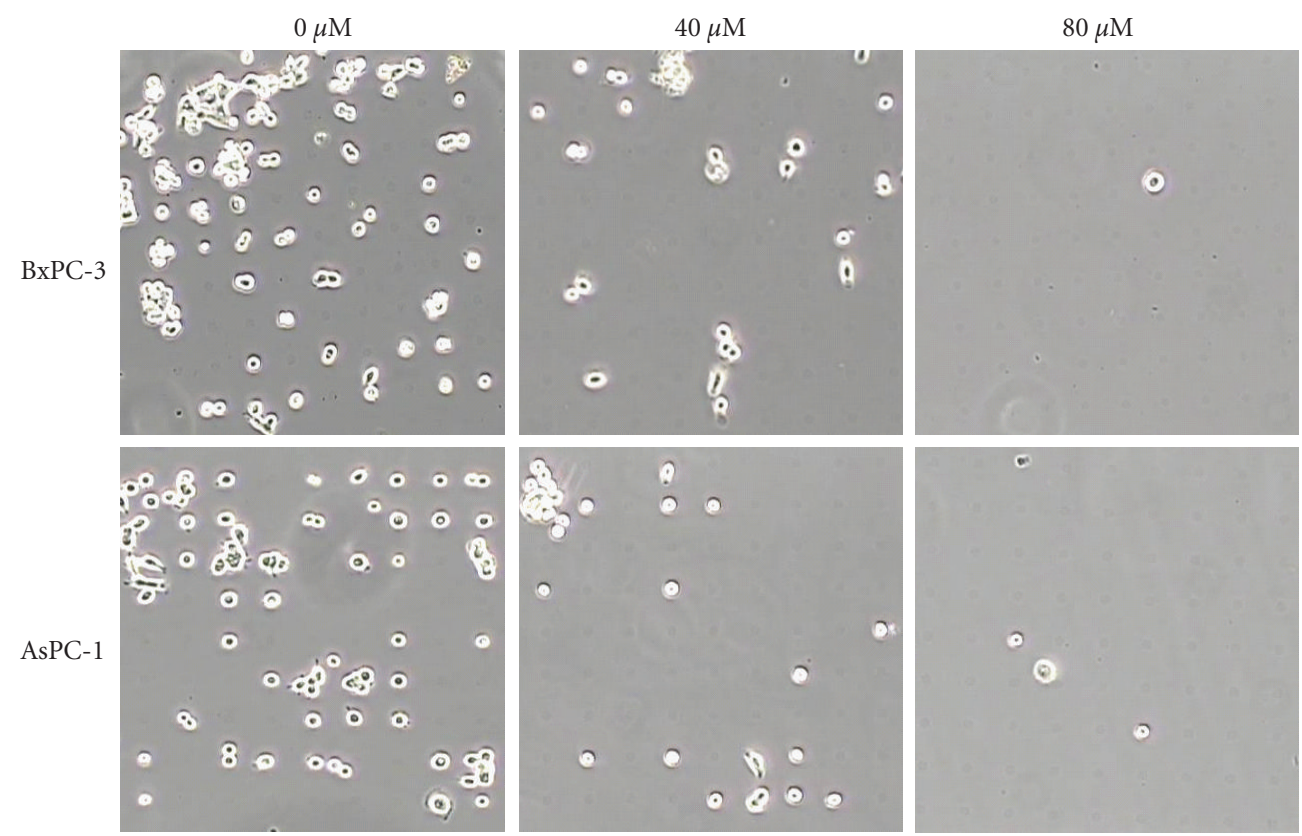

FIGURE 1: EGCG suppresses cell adhesion by micropattern assay. AsPC- 1 and BxPC-3 cells were plated at a density of $0.8 \times 10^{5}$ cells/ml in the presence of indicated doses of EGCG onto micropattern coverslips as described in the Materials and Methods. After 24 hours, cells attaching to single dots were photographed under microscope.

WAKO Chemicals (Osaka, Japan). Alexa fluor 488 goat antirabbit secondary antibody was from Molecular Probes (Eugene, OR, USA).

\subsection{Immunofluorescence Staining and Confocal Microscopy. Cells were plated at a density of $0.8 \times 10^{5}$ cells $/ \mathrm{ml}$ onto coverslips and allowed to attach for 24 hours. After being treated with $100 \mu \mathrm{M}$ EGCG for 6 hours, cells were fixed in $4 \%$ paraformaldehyde for 10 minutes and permeabilized with $0.1 \%$ Triton $\mathrm{X}$ in PBS for 5 minutes. Cells were then washed with PBS, blocked with $2 \%$ bovine serum albumin for 30 minutes, and incubated with p-FAK antibody for 1 hour at room temperature. After washing three times with $0.05 \%$ Triton X in PBS, cells were subsequently incubated with Alexa fluor 488 goat antirabbit secondary antibody for 1 hour at room temperature, and nuclei were stained with Topro 3 before mounting. The slides were viewed using a Zeiss LSM 510 laser confocal microscope (Carl Zeiss, Jena, Germany).}

\section{Results}

3.1. EGCG Suppresses Cell Adhesion on Micropattern Dots. Cell adhesion to other cells or extracellular matrix is of great importance in the development and disease of multicellular organisms. We developed a micropatterned substrate where adhesion of single cells can be observed in order to quantify the effect of EGCG on cell-adhesion capacity (Figure 1). In the absence of EGCG, at a density of $0.8 \times 10^{5}$ cells $/ \mathrm{ml}$, approximately $60 \%$ of dots were occupied with single cells. Cell adhesion was significantly reduced at $40 \mu \mathrm{M}$ EGCG (approximately 20\% of dots were occupied with single cells), and nearly abolished at $80 \mu \mathrm{M}$ EGCG.

3.2. EGCG Inhibits FAK and IGF-IR Activations and the Expression of Target Molecule N-Cadherin in Pancreatic Carcinoma Cells. To understand the molecular mechanism responsible for suppression of cell adhesion by EGCG, we first examine the phosphorylation status of FAK, a key player in cell adhesion [13]. As shown in Figure 2(a), FAK was constitutively activated in pancreatic cancer cells, albeit with lower level in AsPC-1 cells than in BxPC-3 cells. EGCG at $100 \mu \mathrm{M}$ completely abolished phosphorylation of FAK in both cell lines, while expression of total FAK was unchanged. In BxPC-3 cells, which harbor high level of activated FAK, EGCG inhibits the phosphorylation of FAK in a dose-dependent manner (Figure 2(a)). In consistency with western blot results, confocal microscopy analyses confirm that EGCG blocks FAK activation in pancreatic cancer cells (Figure 2(b)).

Because tyrosine phosphorylation of FAK was reported necessary for the interaction of FAK and insulin-like growth factor 1 receptor (IGF-1R) that was important for survival signals in pancreatic cancer cells [5], we next evaluate phosphorylation status of IGF-1R by western blot analysis. Quite similar to FAK, IGF-1R was strongly phosphorylated in BxPC-3 cells, and lower level of phosphorylation was observed in AsPC-1 cells (Figure 2(a)). As expected, $100 \mu \mathrm{M}$ EGCG was enough to abolish phosphorylation of IGF-1R in both cell lines, whereas it had no effect on expression of total IGF-1R. Furthermore, IGF-1R became reactivated at the 20hour time point when EGCG concentration in culture might 


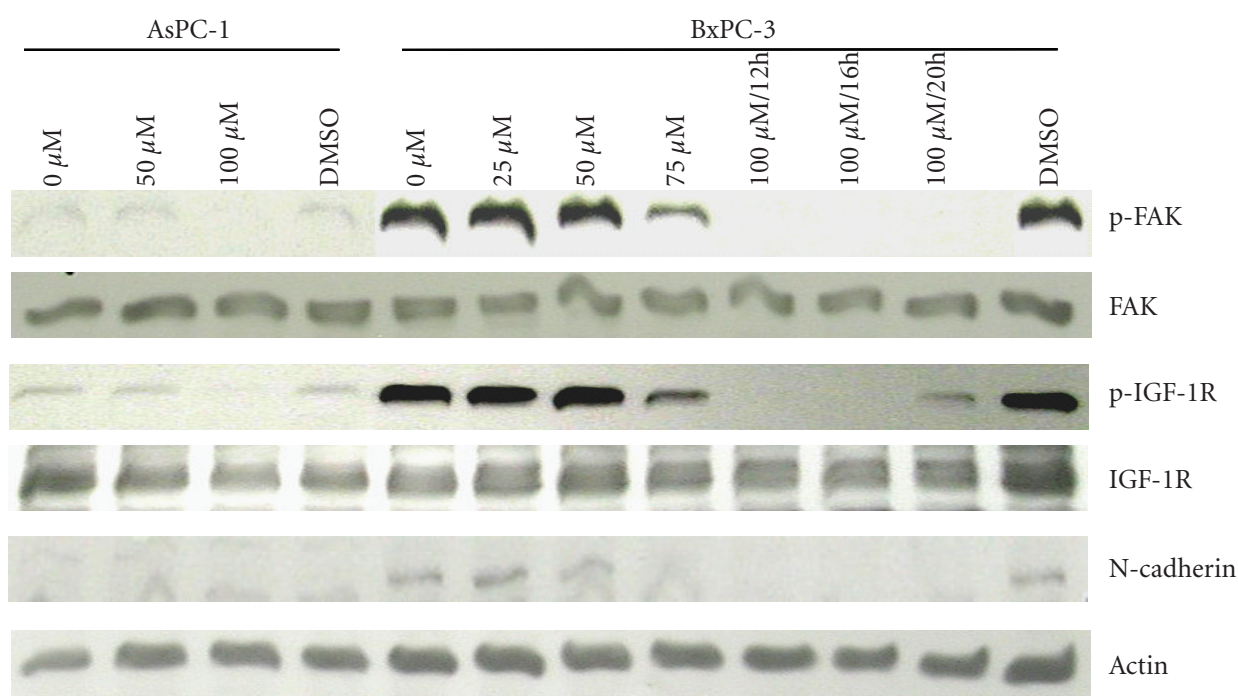

(a)

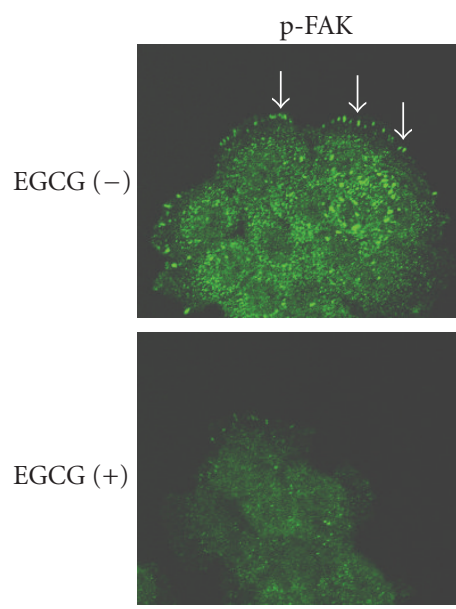

TOPRO-3
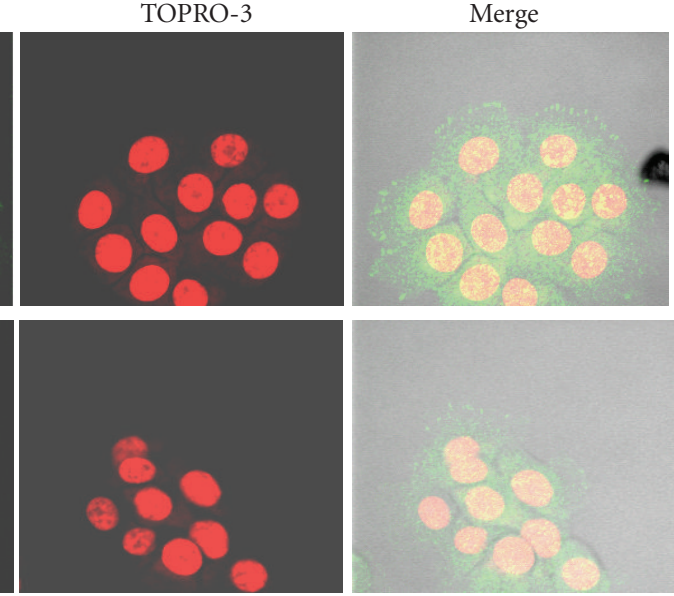

(b)

FIGURE 2: EGCG inhibits FAK activity. (a) Cells were plated at a density of $2 \times 10^{5}$ cells $/ \mathrm{ml}$ in the presence of various concentrations of EGCG for 12 hours, except for BxPC-3 at $100 \mu \mathrm{M}$ for additional 4 or 8 hours. Total cell lysates were subjected to western blot analysis with indicated antibodies. Cells cultured in medium with $0.1 \%$ DMSO were used as a control. (b) Immunofluorescence staining for p-FAK (green) in BxPC-3 cells in the absence or presence of $100 \mu \mathrm{M}$ EGCG, as indicated. Expression of p-FAK (arrows) was significantly reduced in EGCG-treated cells as compared with control cells. Staining with TOPRO-3 (red) visualizes the nuclei.

be decreased so much, indicating that the effect of EGCG on activation of IGF-1R is reversible (Figure $2(\mathrm{a})$ ).

Since FAK upregulates $\mathrm{N}$-cadherin expression in pancreatic cancer cells through p130CAS [14], we ask whether inhibition of FAK by EGCG can downregulate N-cadherin expression. As shown in Figure 2(a), BxPC-3 cells, but not AsPC-1 cells, express $\mathrm{N}$-cadherin. In consistency with FAK activity, $\mathrm{N}$-cadherin expression is abrogated in BxPC-3 cells treated with $100 \mu \mathrm{M}$ EGCG.

3.3. Inhibition of FAK and IGF-IR Activity by EGCG Suppresses Cell Growth in Pancreatic Carcinoma Cells. To determine whether inhibition of FAK and IGF-IR activity by EGCG would lead to cell growth inhibition, TetraColor
ONE cell proliferation assays were performed. As shown in Figure 3(a), EGCG inhibited the cell proliferation in a dosedependent manner, with an $\mathrm{IC}_{50}$ value of approximately $72 \mu \mathrm{M}$ and $64 \mu \mathrm{M}$ for AsPC-1 cells and BxPC-3 cells, respectively. Results were confirmed by trypan blue exclusion assay (data not shown). Under microscope, we also observed that, at low density, all cancer cells were killed by EGCG at $100 \mu \mathrm{M}$ after 48 hours (data not shown). However, as shown in Figure 3(b), the cell growth inhibition by EGCG diminished dramatically with increasing cell densities, similar to phenomena reported in colorectal carcinoma cells [15]. It is noteworthy that at a density of approximately $2 \times 10^{5} \mathrm{cells} / \mathrm{ml}$, EGCG at $100 \mu \mathrm{M}$ is able to suppress more than $50 \%$ cell growth (Figure $3(\mathrm{~b})$ ) and abolish phosphorylation of FAK and IGF-1R as well as $\mathrm{N}$-cadherin 


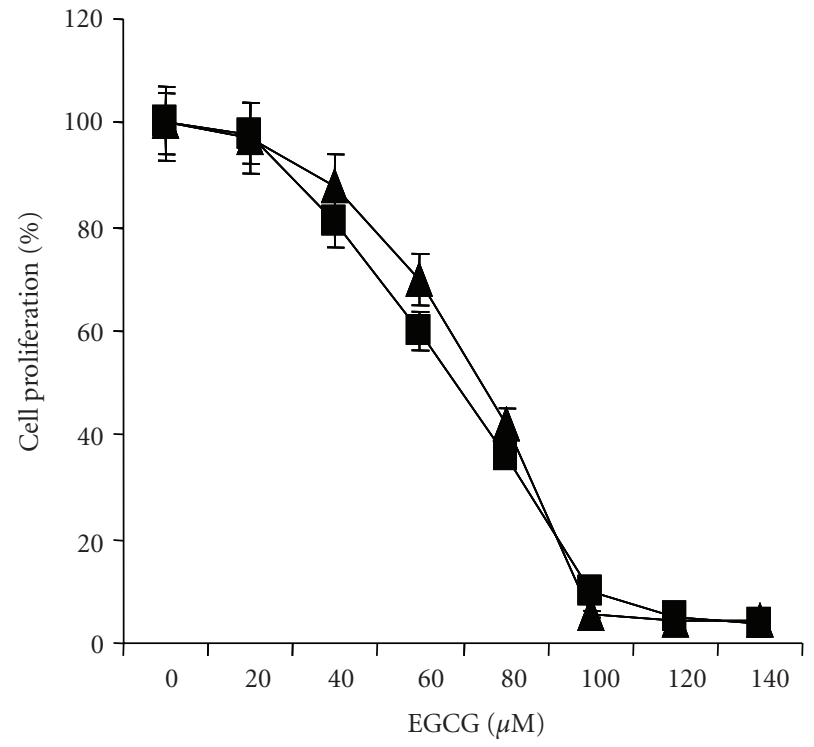

- AsPC-1
- - BхPC-3

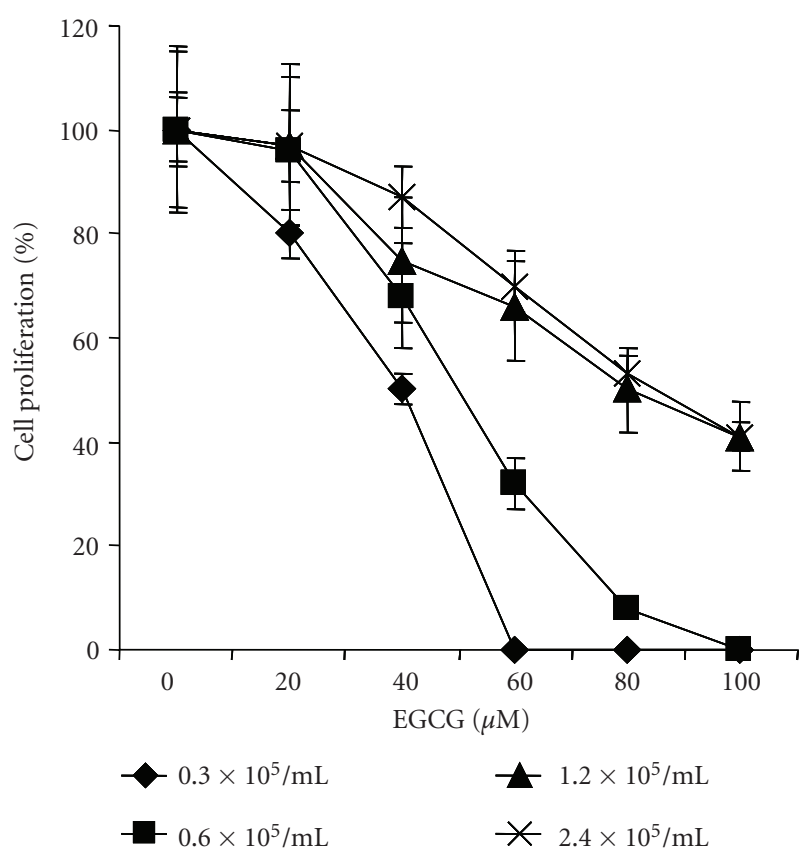

(b)

(a)

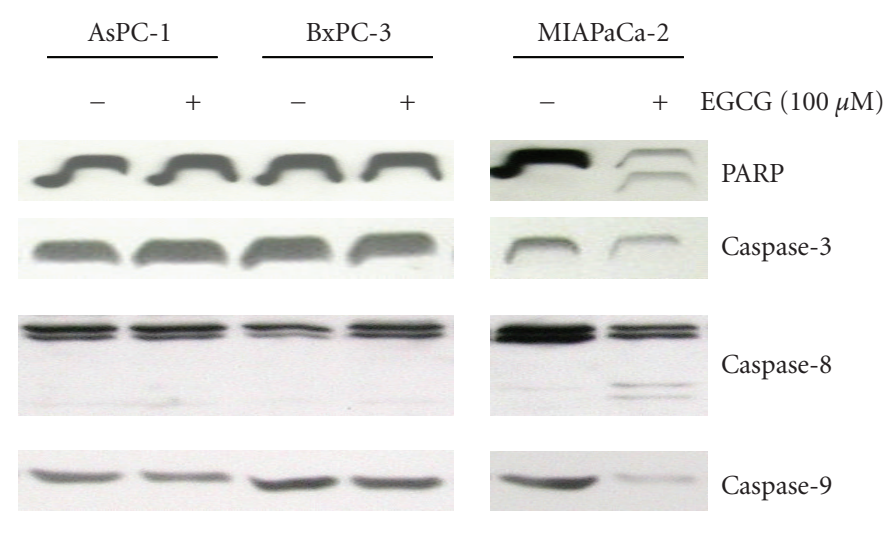

(c)

Figure 3: EGCG suppresses cell proliferation. (a) AsPC-1 and BxPC-3 cells at a density of $0.8 \times 10^{5}$ cells/ml were seeded in quadruplicate in 96-well plates, in $100 \mu \mathrm{l}$ of culture media in the presence of various concentrations of EGCG. After incubation for 48 hours, $10 \mu \mathrm{l}$ of TetraColor ONE reagent was added to each well, and cells were incubated for additional 4 hours. Absorbance at $450 \mathrm{~nm}$ was measured with the Biotrack II plate reader. Results were enumerated as the percentage of the values measured when cells were grown in the absence of EGCG. (b) Cell proliferation assays for BxPC-3 cells at different cell densities. Values were obtained from experiments conducted in triplicate. (c) EGCG fails to cause apoptosis in AsPC-1 and BxPC- 3 cells. Cells were plated at a density of $2 \times 10^{5}$ cells/ml in the presence or absence of $100 \mu \mathrm{M}$ EGCG for 12 hours followed by western blot analysis of total cell lysates using indicated antibodies. MIAPaCa- 2 cells were used as a control of apoptosis induced by EGCG.

expression (Figures 2(a) and 2(b)), but fails to cleave PARP, a sign of caspase-dependent apoptosis, in AsPC-1 and BxPC3 cells (Figure 3(c)). In EGCG-treated BxPC-3 cells, nuclear integrity but not any apoptotic nuclear alterations [16] was visualized by TOPRO-3 staining (Figure 2(b)). In contrast, in MIAPaCa-2 cells used as a control, EGCG induces apoptosis with cleaved PARP accompanied by cleaved caspase 8 [17]. Our results are in consistency with data from Qanungo's study where MIAPaCa-2 cells were sensitive, whereas BxPC3 cells were moderately responsive and AsPC-3 cells were nonresponsive to EGCG-induced PARP cleavage [18].
3.4. MEK1/2 Inhibitor Increases the Antiproliferative Response to EGCG. Since EGCG exhibits antiproliferative effect in AsPC-1 and BxPC-3 cells without evidence of caspasedependent apoptosis, we suspect that EGCG-treated pancreatic cancer cells retain activation of pathways important for cell survival. As shown in Figure 4(a), EGCG up to $100 \mu \mathrm{M}$ fails to inhibit phosphorylation of MAPK and mTOR in both AsPC- 1 and BxPC-3 cells. At first, we were confused by the fact that even though EGCG abolished the phosphorylation of AKT, which was thought to be the main modulator of mTOR activation, it had no effect on phosphorylation 


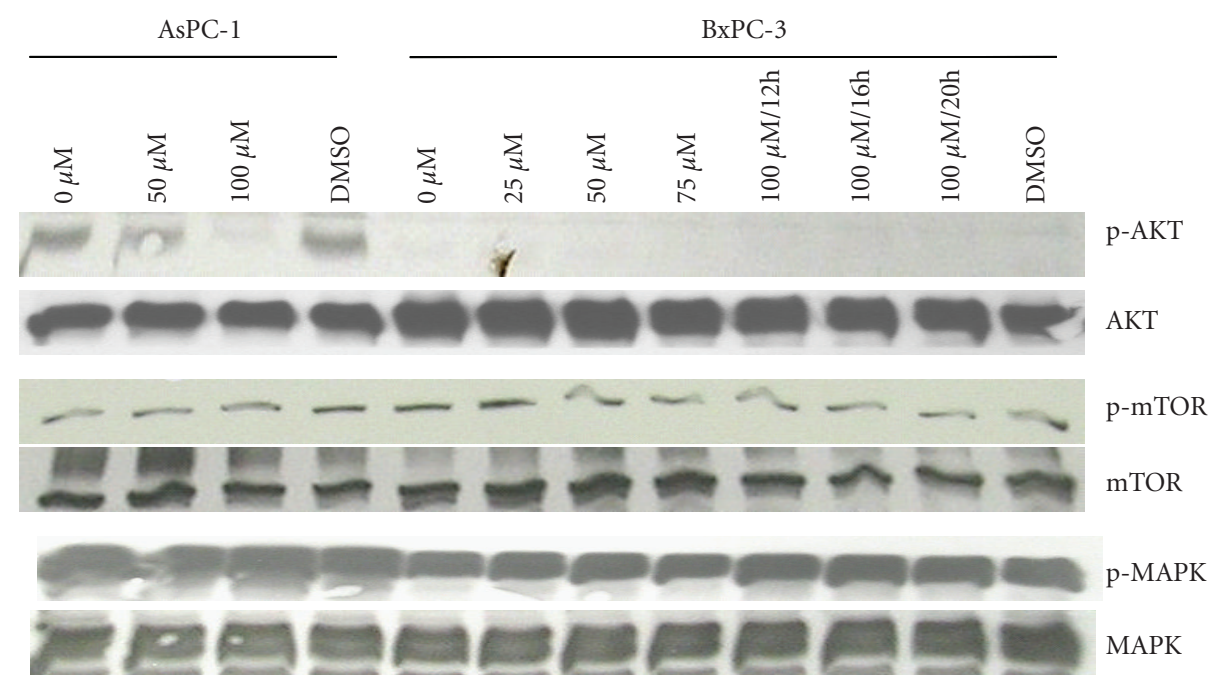

(a)
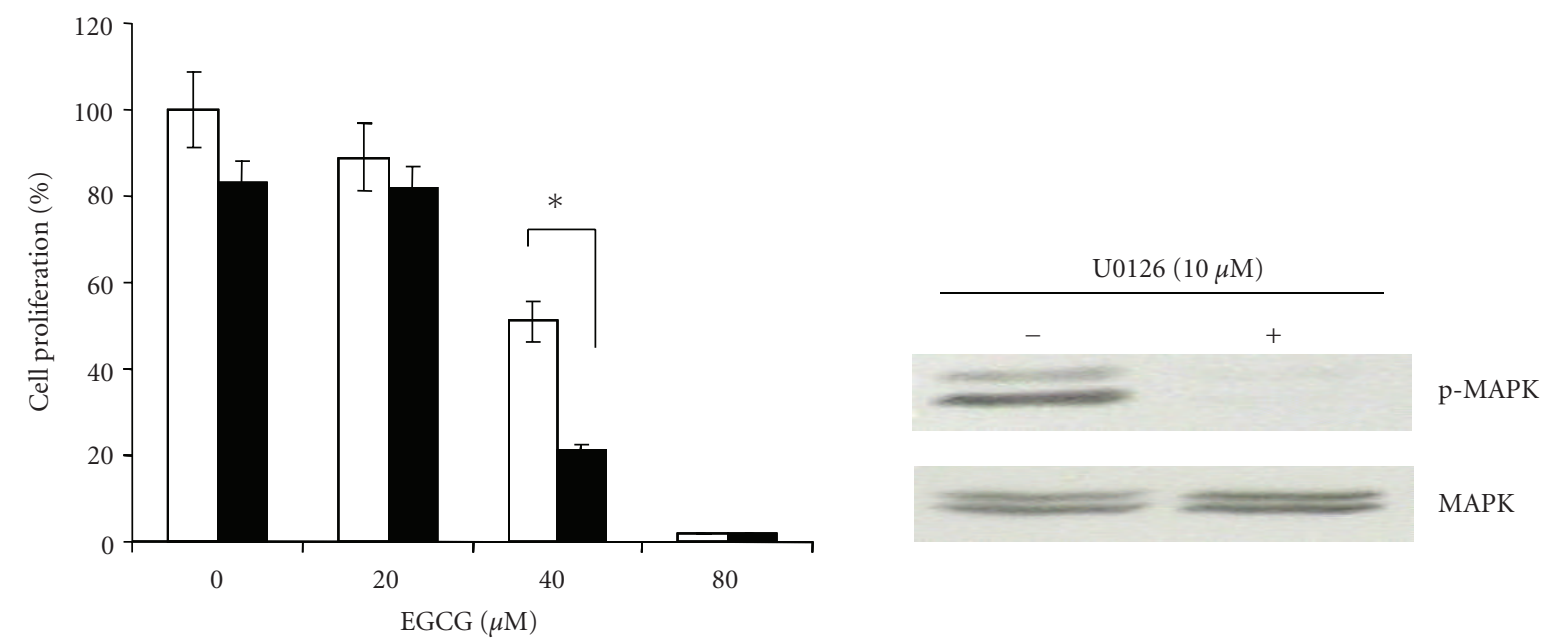

U0126 (-)

U0126 (+)

(b)

FIgUre 4: (A) EGCG fails to inhibit phosphorylation of MAPK and mTOR in AsPC-1 and BxPC-3 cells. Cells were plated at a density of $2 \times 10^{5}$ cells $/ \mathrm{ml}$ in the presence of various concentrations of EGCG for 12 hours, except for BxPC-3 at $100 \mu \mathrm{M}$ for additional 4 or 8 hours. Total cell lysates were subjected to western blot analysis. (b) MAPK inhibitor enhances EGCG-induced antiproliferative response in BxPC-3 cells. Cells were plated at a density of $0.6 \times 10^{5}$ cells $/ \mathrm{ml}$ in 24 -well dishes with different concentrations of EGCG for 48 hours in the presence or absence of $10 \mu \mathrm{M}$ U0126 (MEK1/2 inhibitor). Cells were detached by trypsin, stained with trypan blue and counted manually using a hemacytometer. Values were obtained from experiments conducted in triplicate. Asterisk indicates significant difference. Inhibition of phosphorylation of MAPK by U0126 at $10 \mu \mathrm{M}$ was confirmed with western blot analysis.

of mTOR in AsPC-1 cells (Figure 4(a)); however, very recently, Stephan et al. have clearly demonstrated that $\mathrm{PKC} \delta$ but not AKT acts upstream of mTOR and that mTOR phosphorylation was $\mathrm{PKC} \delta$ dependent in AsPC-1 cells [19]. It has been also reported that inhibition of migration in bladder carcinoma cells associated with AKT but not MAPK signaling [20].

We next want to know whether inhibition of MAPK activity by an inhibitor can enhance antiproliferative response of pancreatic cancer cells to EGCG. As expected,
MAPK inhibitor potentiated the EGCG-induced antiproliferative response in BxPC-3 cells (Figure 4(b)). A similar result was observed in AsPC-1 cells (data not shown).

\section{Discussion}

Advances in pancreatic carcinogenesis have provided more novel promising targets for prevention and treatment. Among them, upregulation of $\mathrm{N}$-cadherin expression plays a key role in tumor progression and metastasis [21]. Even 
though it was reported that EGCG downregulated Ncadherin expression and suppressed migration of bladder carcinoma cells [20], the molecular mechanism underlying downregulation of $\mathrm{N}$-cadherin expression was not addressed. Recently, Shintani group have demonstrated that FAK upregulated N-cadherin expression in pancreatic cancer cells [14]. In agreement with their findings, we have shown here for the first time that EGCG abolished N-cadherin expression in pancreatic cancer cells via inhibition of FAK activation (Figure 2). The inhibition was accompanied by blocking activation of IGF-1R, which interacts with FAK to provide survival signals in pancreatic cancer cells [5]. Indeed, EGCG was shown to be a highly potent inhibitor of IGF-1R tyrosine kinase activity [22]. On the other hand, in human colon carcinoma cells, inhibition of FAK activity by EGCG was reported to alter invasive phenotype [23]. We have further observed inhibition of FAK activity by EGCG in 2 prostate cancer cell lines PC-3 and DU145 (data not shown). Whether inhibition of FAK by EGCG in pancreatic cancer cells can actually prevent metastasis requires further animal study. However, at least, the inhibition of both FAK and IGF-1R could lead to cell growth inhibition (Figures 3(a) and 3(b)). The fact that cell growth inhibition depends on cell density, where cell population with low density but not that with high density could be killed completely by EGCG (Figure 3(b)), predicts that metastasis prevention by EGCG can be achieved a only if it is used at very early stage, before metastasis has been set in by large numbers of metastatic cancer cells.

Clinical trials of EGCG in patients with cancers confirmed that the substance was well tolerated and supported a potential role for EGCG in the treatment and prevention of cancer $[24,25]$. Due to a relatively short half life plus low oral bioavailability, high concentrations of EGCG are unattainable in vivo, particularly in plasma, even if people consume an excess of EGCG. However, it may be feasible to achieve therapeutic dosages at relatively low dosages of prodrug Petacetate-EGCG which is readily taken up by tumor cells and converted to EGCG [26]. In mice, EGCG might be effectively delivered locally up to $200 \mu \mathrm{M}$ to eradicate aggressive, metastatic tumors [27].

With respect to therapeutic aspect, our findings do not support the application of EGCG alone for pancreatic cancer, because of its limited effect on cell growth inhibition, without evidence of apoptosis (Figures 3(b) and $3(c)$ ). Instead, combination of EGCG with other inhibitors such as MAPK inhibitor should be tested for this dismal cancer, since MAPK inhibitor enhanced the EGCG-induced antiproliferative response in cancer cell lines (Figure 4(b)). We observed that EGCG at $200 \mu \mathrm{M}$ had no significant effect on the healthy human peripheral blood mononuclear cells and human lung fibroblast cell line WI-38 (data not shown), indicating specific toxicity of EGCG towards cancer cells. Pancreatic cancer is an aggressive disease with aberrant activations of numerous signaling pathways. Among them, activation of MAPK by KRAS or BRAF mutation promotes the survival and growth of cancer cells [28, 29]. A phase I trial of BAY 43-9006/sorafenib, a specific inhibitor of RAF, in combination with gemcitabine for patients with pancreatic cancer indicated that the therapy was well tolerated [30].
Together, current findings warrant a combination of MAPK inhibitor such as sorafenib and EGCG for pancreatic cancer.

This work was presented in the International Conference on the Development of Biomedical Engineering in Vietnam [31].

\section{References}

[1] D. Maxwell Parkin, F. Bray, J. Ferlay, and P. Pisani, "Estimating the world cancer burden: Globocan 2000," International Journal of Cancer, vol. 94, no. 2, pp. 153-156, 2001.

[2] T. P. Hecker and C. L. Gladson, "Focal adhesion kinase in cancer," Frontiers in Bioscience, vol. 8, pp. s705-s714, 2003.

[3] D. J. Sieg, C. R. Hauck, D. Ilic et al., "FAK integrates growthfactor and integrin signals to promote cell migration," Nature Cell Biology, vol. 2, no. 5, pp. 249-256, 2000.

[4] V. M. Golubovskaya, S. Gross, A. S. Kaur et al., "Simultaneous inhibition of focal adhesion kinase and Src enhances detachment and apoptosis in colon cancer cell lines," Molecular Cancer Research, vol. 1, no. 10, pp. 755-764, 2003.

[5] W. Liu, D. A. Bloom, W. G. Cance, E. V. Kurenova, V. M. Golubovskaya, and S. N. Hochwald, "FAK and IGFIR interact to provide survival signals in human pancreatic adenocarcinoma cells," Carcinogenesis, vol. 29, no. 6, pp. 10961107, 2008.

[6] T. W. Bauer, R. J. Somcio, F. Fan et al., "Regulatory role of c-Met in insulin-like growth factor-I receptor-mediated migration and invasion of human pancreatic carcinoma cells," Molecular Cancer Therapeutics, vol. 5, no. 7, pp. 1676-1682, 2006.

[7] A. Hakam, Q. Fang, R. Karl, and D. Coppola, "Coexpression of IGF-1R and c-Src proteins in human pancreatic ductal adenocarcinoma," Digestive Diseases and Sciences, vol. 48, no. 10, pp. 1972-1978, 2003.

[8] C. Moser, P. Schachtschneider, S. A. Lang et al., "Inhibition of insulin-like growth factor-I receptor (IGF-IR) using NVPAEW541, a small molecule kinase inhibitor, reduces orthotopic pancreatic cancer growth and angiogenesis," European Journal of Cancer, vol. 44, no. 11, pp. 1577-1586, 2008.

[9] N. Watanabe, M. Takaoka, K. Sakurama et al., "Dual tyrosine kinase inhibitor for focal adhesion kinase and insulinlike growth factor-I receptor exhibits anticancer effect in esophageal adenocarcinoma in vitro and in vivo," Clinical Cancer Research, vol. 14, no. 14, pp. 4631-4639, 2008.

[10] TA. J. Liu, T. LaFortune, T. Honda et al., "Inhibition of both focal adhesion kinase and insulin-like growth factor-I receptor kinase suppresses glioma proliferation in vitro and in vivo," Molecular Cancer Therapeutics, vol. 6, no. 4, pp. 1357-1367, 2007.

[11] N. Faucheux, R. Schweiss, K. Lützow, C. Werner, and T. Groth, "Self-assembled monolayers with different terminating groups as model substrates for cell adhesion studies," Biomaterials, vol. 25, no. 14, pp. 2721-2730, 2004.

[12] H. A. Vu, P. T. Xinh, M. Masuda et al., "FLT3 is fused to ETV6 in a myeloproliferative disorder with hypereosinophilia and a t(12;13)(p13;q12) translocation," Leukemia, vol. 20, no. 8, pp. 1414-1421, 2006.

[13] B. D. Cox, M. Natarajan, M. R. Stettner, and C. L. Gladson, "New concepts regarding focal adhesion kinase promotion of cell migration and proliferation," Journal of Cellular Biochemistry, vol. 99, no. 1, pp. 35-52, 2006. 
[14] Y. Shintani, Y. Fukumoto, N. Chaika, R. Svoboda, M. J. Wheelock, and K. R. Johnson, "Collagen I-mediated upregulation of $\mathrm{N}$-cadherin requires cooperative signals from integrins and discoidin domain receptor," Journal of Cell Biology, vol. 180, no. 6, pp. 1277-1289, 2008.

[15] H. Inaba, Y. Nagaoka, Y. Kushima et al., "Comparative examination of anti-proliferative activities of (-)-epigallocatechin gallate and (-)-epigallocatechin against HCT116 colorectal carcinoma cells," Biological and Pharmaceutical Bulletin, vol. 31, no. 1, pp. 79-84, 2008.

[16] B. Pyrzynska, M. Serrano, C. Martínez-A, and B. Kaminska, "Tumor suppressor p53 mediates apoptotic cell death triggered by cyclosporin A," Journal of Biological Chemistry, vol. 277, no. 16, pp. 14102-14108, 2002.

[17] A. Basu and S. Haldar, "Combinatorial effect of epigallocatechin-3-gallate and TRAIL on pancreatic cancer cell death," International Journal of Oncology, vol. 34, no. 1, pp. 281-286, 2009.

[18] S. Qanungo, M. Das, S. Haldar, and A. Basu, "Epigallocatechin-3-gallate induces mitochondrial membrane depolarization and caspase-dependent apoptosis in pancreatic cancer cells," Carcinogenesis, vol. 26, no. 5, pp. 958-967, 2005.

[19] S. Stephan, A. Musacchio, and Y. Hagos, "Insulin receptor substrate-2 mediated insulin-like growth factor-I receptor overexpression in pancreatic adenocarcinoma through protein kinase C $\delta$," Cancer Research, vol. 69, no. 4, pp. 1350-1357, 2009.

[20] K. M. Rieger-Christ, R. Hanley, C. Lodowsky et al., "The green tea compound, (-)-epigallocatechin-3-gallate downregulates $\mathrm{N}$-cadherin and suppresses migration of bladder carcinoma cells," Journal of Cellular Biochemistry, vol. 102, no. 2, pp. 377388, 2007.

[21] Y. Shintani, M. A. Hollingsworth, M. J. Wheelock, and K. R. Johnson, "Collagen I promotes metastasis in pancreatic cancer by activating c-Jun NH-terminal kinase 1 and up-regulating N-cadherin expression," Cancer Research, vol. 66, no. 24, pp. 11745-11753, 2006.

[22] M. Li, Z. He, S. Ermakova et al., "Direct inhibition of insulin-like growth factor-I receptor kinase activity by (-)epigallocatechin-3-gallate regulates cell transformation," Cancer Epidemiology Biomarkers and Prevention, vol. 16, no. 3, pp. 598-605, 2007.

[23] M. J. Weyant, A. M. Carothers, A. J. Dannenberg, and M. M. Bertagnolli, " $(+)$-catechin inhibits intestinal tumor formation and suppresses focal adhesion kinase activation in the Min/+ mouse," Cancer Research, vol. 61, no. 1, pp. 118-125, 2001.

[24] J. McLarty, R. L. H. Bigelow, M. Smith, D. Elmajian, M. Ankem, and J. A. Cardelli, "Tea polyphenols decrease serum levels of prostate-specific antigen, hepatocyte growth factor, and vascular endothelial growth factor in prostate cancer patients and inhibit production of hepatocyte growth factor and vascular endothelial growth factor in vitro," Cancer Prevention Research, vol. 2, no. 7, pp. 673-682, 2009.

[25] T. D. Shanafelt, T. G. Call, C. S. Zent et al., "Phase I trial of daily oral polyphenon $\mathrm{E}$ in patients with asymptomatic rai stage 0 to II chronic lymphocytic leukemia," Journal of Clinical Oncology, vol. 27, no. 23, pp. 3808-3814, 2009.

[26] K. R. Landis-Piwowar, C. Huo, DI. Chen et al., "A novel prodrug of the green tea polyphenol (-)-epigallocatechin-3gallate as a potential anticancer agent," Cancer Research, vol. 67, no. 9, pp. 4303-4310, 2007.

[27] M. E. Stearns, M. D. Amatangelo, D. Varma, C. Sell, and S. M. Goodyear, "Combination therapy with epigallocatechin-3gallate and doxorubicin in human prostate tumor modeling studies: inhibition of metastatic tumor growth in severe combined immunodeficiency mice," American Journal of Pathology, vol. 177, no. 6, pp. 3169-3179, 2010.

[28] C. Almoguera, D. Shibata, K. Forrester, J. Martin, N. Arnheim, and M. Perucho, "Most human carcinomas of the exocrine pancreas contain mutant c-K-ras genes," Cell, vol. 53, no. 4, pp. 549-554, 1988.

[29] E. S. Calhoun, J. B. Jones, R. Ashfaq et al., "BRAF and FBXW7 (CDC4, FBW7, AGO, SEL10) mutations in distinct subsets of pancreatic cancer: potential therapeutic targets," American Journal of Pathology, vol. 163, no. 4, pp. 1255-1260, 2003.

[30] L. L. Siu, A. Awada, C. H. Takimoto et al., "Phase I trial of sorafenib and gemcitabine in advanced solid tumors with an expanded cohort in advanced pancreatic cancer," Clinical Cancer Research, vol. 12, no. 1, pp. 144-151, 2006.

[31] H. A. Vu, Y. Beppu, H. T. Chi et al., "Green tea epigalocatechin gallate exhibits anticancer effect in human pancreatic carcinoma cells via inhibition of both FAK and IGF-1R," IFMBE Proceedings, vol. 27, pp. 223-226, 2010. 


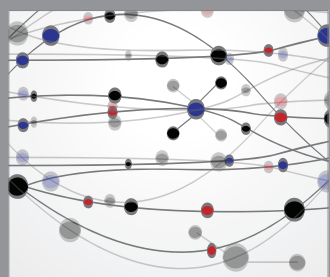

The Scientific World Journal
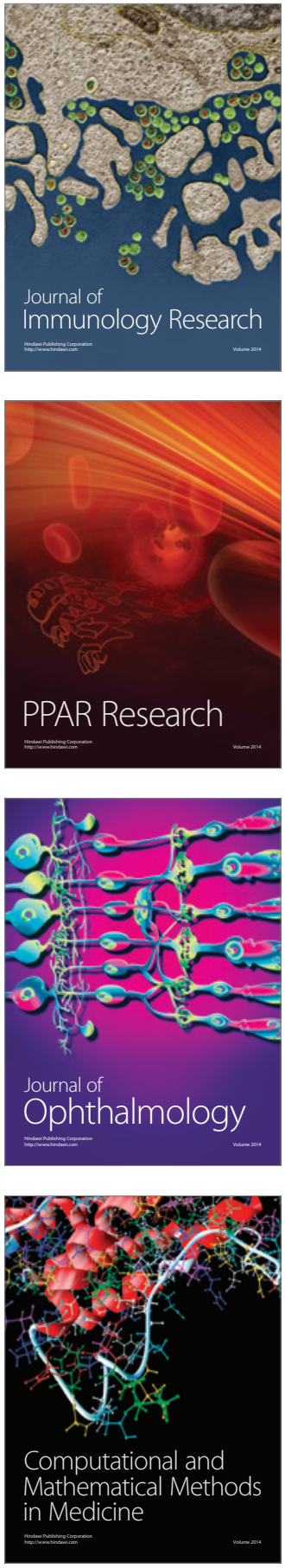

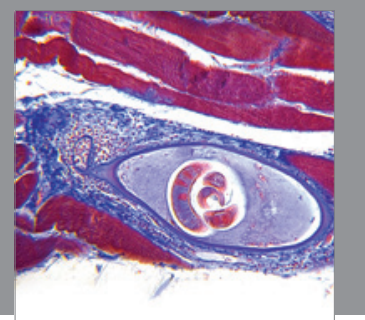

Gastroenterology

Research and Practice
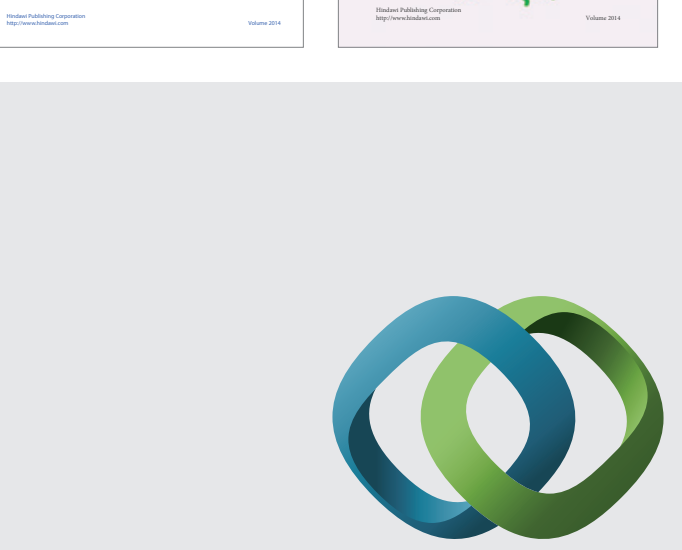

\section{Hindawi}

Submit your manuscripts at

http://www.hindawi.com
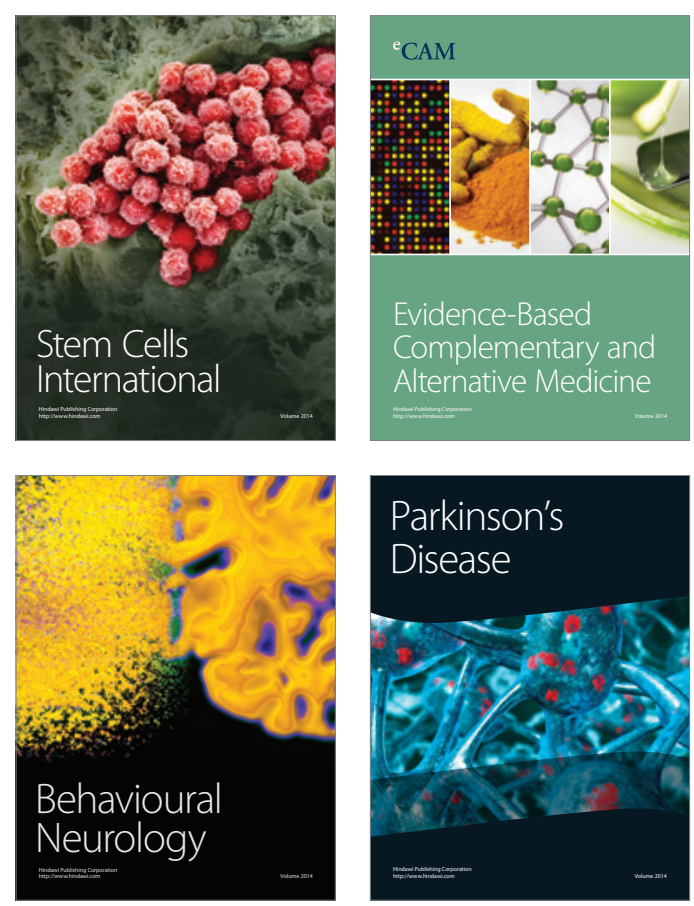

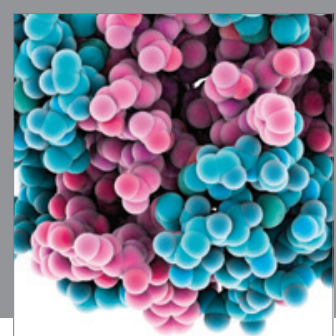

Journal of
Diabetes Research

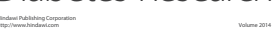

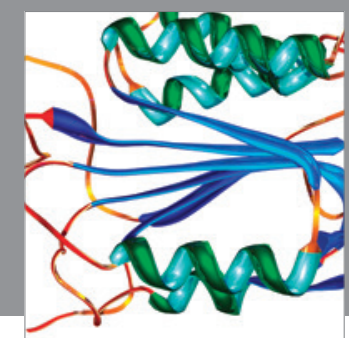

Disease Markers
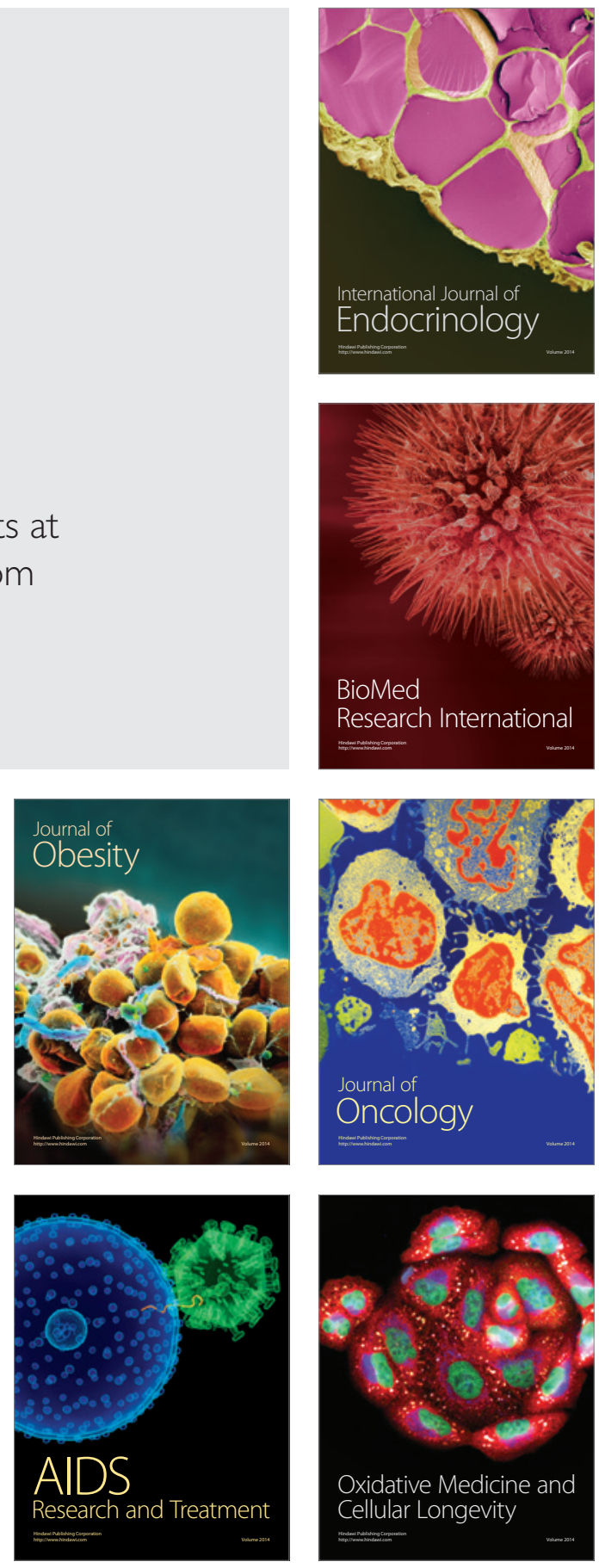